\title{
BCG vaccination for COVID-19: Evidence-based medicine approach
}

\author{
Mina Kelleni ${ }^{1}$ \\ ${ }^{1}$ Minia University
}

June 12, 2020

Multiple reports all over the world are hypothesizing a possible relationship between BCG vaccination, and a lesser incidence of and mortality from COVID-19 ${ }^{1}$. China is considered as one of the heavily affected countries and it was the first to report COVID-19, the author will examine this hypothesis using China as an example.

According to the WHO global tuberculosis report $2019^{2}$, it's stated that in 2018, among 180 countries for which data were collected, 153 countries have properly reported administration of BCG vaccination as a standard part of childhood immunization programmes, of which 113 reported coverage of [?] $90 \%$ (Figure 1). In the same report, China has accomplished a $99 \%$ BCG vaccination coverage, to be considered as one of the top countries providing this coverage rate all over the world. Moreover, the reported estimates for the global BCG coverage according to the WHO monitoring system for vaccine-preventable diseases has revealed that BCG vaccination coverage in China to be either $99 \%$ or $100 \%$ for the years 2009 to $2018^{3}$. Thus, we may reasonably consider that all children, currently 10 years old or younger and living in China, are efficiently covered and immunized by BCG vaccination.

Interestingly, as of the $11^{\text {th }}$ of February, 2020 the Chinese Center for Disease Control and Prevention has reported 416 laboratory-confirmed pediatric cases; aged 0-9 years ${ }^{4}$. Moreover, another study, published about one month later, has researched 2143 pediatric Chinese patients who were both laboratory-confirmed as well as suspected cases, and concluded that children at all ages appeared susceptible to COVID-19 and it's also reported that the proportion of severe and critical cases was $10.6 \%, 7.3 \%, 4.2 \%$ for the age group of $<1,1-5,6-10$, respectively. Noteworthy, this study has suggested that the majority of COVID-19 pediatric patients were less severe in their medical condition than adults' cases because children are usually well cared for at home and might have relatively less opportunities to expose themselves to SARS CoV-25 .

Thus, basing on the above analysis, the author suggests that stating that BCG vaccination could prevent COVID-19 infection should be considered as a myth. Moreover, the author considers stating that countries which have adopted childhood BCG vaccination might be the reason for a lower morbidity or mortality of COVID-19 for adults immunized at birth, should be also considered as another myth, as revealed by the incidence of adults' morbidity and mortality rates reported in China as well as several other countries that have adopted childhood BCG vaccination. However, BCG vaccination given once a month for three consecutive months has previously showed a benefit against adult upper respiratory tract infections in the elderly $^{6}$ and it might prove the same benefits against COVID-19 if administered to unimmunized adults or re-administered to previously immunized adults waiting for the results of the undergoing clinical trials ${ }^{7}$. On the other hand, BCG vaccination might or might not have some ameliorative benefits for immunized children affected by COVID-19 and this suggested positive association is highly unlikely to be properly proved because of multiple confounding factors. Finally, please keep and remain vigilant till the world finds a cure for COVID-19, this is an evidence-based medicine piece of advice.

Funding: 
None

Conflict of interests:

None

Financial disclosure:

None

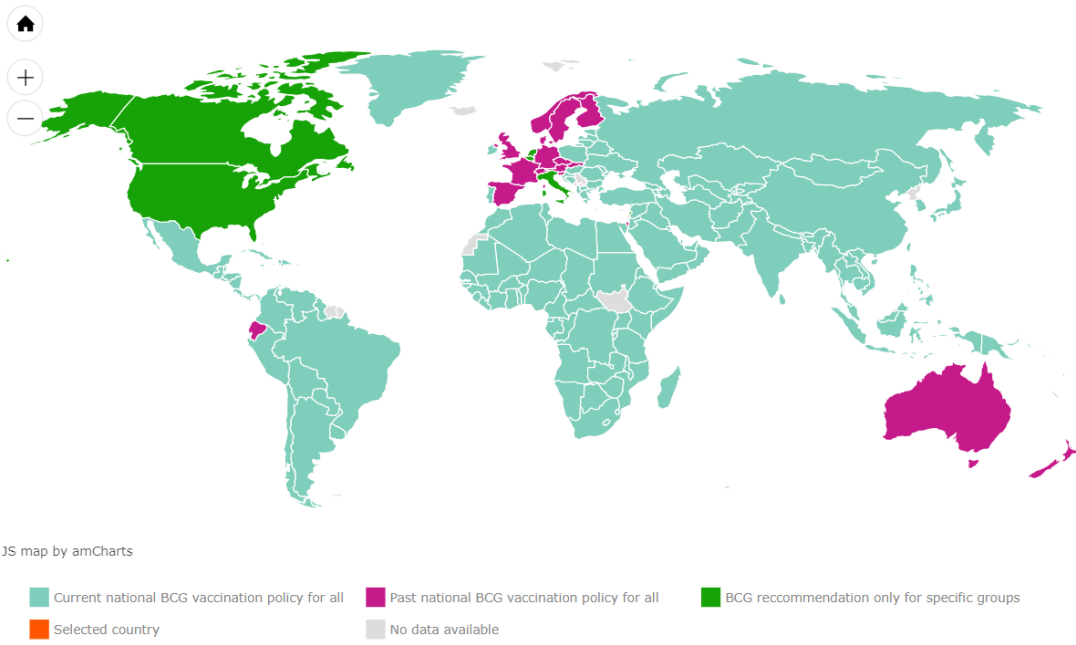

Fig (1): GLOBAL BCG VACCINATION POLICIES AND PRACTICES

Source: The BCG World Atlas 2nd Edition, http://www.bcgatlas.org/, accessed 5 May, 2020.

Conflict of interests:

None

References:

1. Redelman-Sidi G. Could BCG be used to protect against COVID-19? Nature Reviews Urology 2020 doi: $10.1038 / \mathrm{s} 41585-020-0325-9$

2. WHO. Global tuberculosis report 2019 2019, October 17 [Available from: https://apps.who.int/iris/bitstream/handle/10665/329368/9789241565714-eng.pdf?ua=1.

3. WHO. WHO vaccine-preventable diseases: monitoring system. 2019 global summary 2019, December 10 [Available from: https://apps.who.int/immunization_monitoring/globalsummary/coverages?c=CHN.

4. Choi SH, Kim HW, Kang JM, et al. Epidemiology and Clinical Features of Coronavirus disease 2019 in Children. Clin Exp Pediatr 2020 doi: 10.3345/cep.2020.00535 [published Online First: 2020/04/07]

5. Dong Y, Mo X, Hu Y, et al. Epidemiological Characteristics of 2143 Pediatric Patients With 2019 Coronavirus Disease in China.Pediatrics 2020:e20200702. doi: 10.1542/peds.2020-0702

6. Wardhana, Datau EA, Sultana A, et al. The efficacy of Bacillus Calmette-Guerin vaccinations for the prevention of acute upper respiratory tract infection in the elderly. Acta Med Indones2011;43(3):185-90. [published Online First: 2011/10/08]

\begin{tabular}{lllll|l|l} 
7. ClinicalTrials.gov. & 7 Studies found for:
\end{tabular} May 2 [Available from: https://clinicaltrials.gov/ct2/results?cond=COVID$19 \&$ term $=$ BCG\&cntry $=\&$ state $=\&$ city $=\&$ dist $=\&$ Search $=$ Search. 